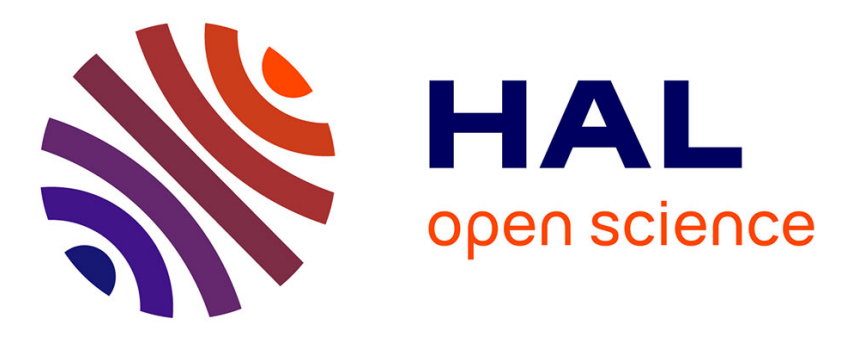

\title{
Strategic Dominance and Dynamic Programming for Multi-agent Planning - Application to the Multi-Robot Box-pushing Problem
}

Mohamed Amine Hamila, Emmanuelle Grislin-Le Strugeon, René Mandiau, Abdel-Illah Mouaddib

\section{To cite this version:}

Mohamed Amine Hamila, Emmanuelle Grislin-Le Strugeon, René Mandiau, Abdel-Illah Mouaddib. Strategic Dominance and Dynamic Programming for Multi-agent Planning - Application to the Multi-Robot Box-pushing Problem. International Conference on Agents and Artificial Intelligence (ICAART), 2012, Algarve, Portugal. p.91-97. hal-00971770

\author{
HAL Id: hal-00971770 \\ https://hal.science/hal-00971770
}

Submitted on 2 Dec 2014

HAL is a multi-disciplinary open access archive for the deposit and dissemination of scientific research documents, whether they are published or not. The documents may come from teaching and research institutions in France or abroad, or from public or private research centers.
L'archive ouverte pluridisciplinaire HAL, est destinée au dépôt et à la diffusion de documents scientifiques de niveau recherche, publiés ou non, émanant des établissements d'enseignement et de recherche français ou étrangers, des laboratoires publics ou privés. 


\title{
[ \\ STRATEGIC DOMINANCE AND DYNAMIC PROGRAMMING FOR MULTI-AGENT PLANNING: APPLICATION TO THE MULTI-ROBOT BOX-PUSHING PROBLEM
}

\author{
Mohamed Amine Hamila ${ }^{2}$, Emmanuelle Grislin-Le Strugeon ${ }^{2}$, Rene Mandiau ${ }^{2}$, Abdel-Illah Mouaddib ${ }^{4}$ \\ ${ }^{2}$ LAMIH, Universite de Valenciennes, France \\ ${ }^{4}$ GREYC, Universite de Caen Basse-Normandie, France \\ \{mohamed.hamila,emmanuelle.grislin,rene.mandiau\}@univ-valenciennes.fr, mouaddib@info.unicaen.fr
}

Keywords: Multi-agent Planning; Coordination; Stochastic Games; Markov processes

Abstract: $\quad$ This paper presents a planning approach for a multi-agent coordination problem in a dynamic environment. We introduce the algorithm SGInfiniteVI, allowing to apply some theories related to the engineering of multi-agent systems and designed to solve stochastic games. In order to limit the decision complexity and so decreasing the used resources (memory and processor-time), our approach relies on reducing the number of joint-action at each step decision. A scenario of multi-robot Box-pushing is used as a platform to evaluate and validate our approach. We show that only weakly dominated actions can improve the resolution process, despite a slight deterioration of the solution quality due to information loss. 


\section{Introduction}

Many daily situations involve a decision making: for example an air-traffic controller has to assign landingarea and time slots to planes, or a taxi company that has some transportation tasks to be carried out. Intelligent agents can aid in this decision-making process. In this paper, we address the problem of collision-free paths for multiple agents sharing and moving in the same environment.

The objective of this work is to propose an efficient answer to such coordination problems. One answer is to consider stochastic games, since they provide a powerful framework for modeling multi-agent interactions. Stochastic games were first studied as an extension of matrix games (Neumann and Morgenstern, 1944) to multiple states. They are also seen as a generalization of Markov decision process (MDP) (Puterman, 2005) to several agents. The Nash equilibrium (Nash, 1950) is the most commonly-used solution concept, intuitively defined as a particular behavior for all agents, where each agent acts optimally with regard to the others' behavior.

This work aims to improve the performance of a previous algorithm, the SGInfiniteVI (Hamila et al., 2010), designed to solve stochastic games. The latter allowed finding a decentralized policy actions, based on the dynamic programming technique and Nash equilibria. However, the computed solution is made through a comprehensive process, thereby limiting the dimensions of the addressed problem.

Our contribution is mainly threefold; firstly, we present an exact algorithm for the elimination of weakly/strictly dominated strategies. Secondly, we have incorporated this technique into the algorithm SGInfiniteVI, in order to simplify the decision problems and accelerate the resolution process. Thirdly, we proposed an experimental approach for the evaluation of the resulting new algorithm and it is performed in two stages; (1) numerical evaluation: attempts to compare the effect of the elimination of weakly and strictly dominated strategies, on the used resources, (2) Behavioral evaluation: checks the impact of the chosen strategy on the solution quality.

The paper has been organized as follows. Section 2 recalls some definitions of stochastic games. Section 3 describes the algorithm SGInfiniteVI, the process improvement and shows how to apply on a grid-world game. Results are presented and discussed in section 4 and finally we conclude in section 5.

\section{Background}

In this section, we present on one hand an introduction to the model of stochastic games and on the other hand some of its crucial aspects.

\subsection{Definitions and concepts}

Stochastic Games (SG) (Shoham et al., 2003; Hansen et al., 2004) are defined by the tuple: $<A g,\left\{A_{i}: i=1 \ldots|A g|\right\},\left\{R_{i}: i=1 \ldots|A g|\right\}, S, T>$

- $A g:$ is the finite set of agents.

- $A_{i}$ : is the finite set of actions (or pure strategies) available to agent $i(i \in A g)$.

- $R_{i}$ : is the immediate reward function of agent $i, R_{i}(a) \rightarrow \mathbb{R}$, where $a$ is the joint-action defined as $a \in \times_{i \in A g} A_{i}$ and is given by $a=\left\langle a_{1}, \ldots, a_{|A g|}\right\rangle$.

- $S$ : is the finite set of environment states.

- $T$ : is the stochastic transition function,

$T: S \times A \times S \rightarrow[0,1]$, indicating the probability of moving from a state $s \in S$ to a state $s^{\prime} \in S$ by running the joint-action $a$.

The particularity of stochastic games is that each state $s$ can be considered as a matrix game $M(s)$. At each step of the game, the agents observe their environment, simultaneously choose actions and receive rewards. The environment transitions stochastically into a different state $M\left(s^{\prime}\right)$ with a probability $P\left(s^{\prime} \mid s, a\right)$ and the above process repeats. The goal for each agent is to maximize the expected sum of rewards it receives during the game.

\subsection{Equilibrium in stochastic games}

Stochastic games have reward functions which can be different for every agent. In certain cases, it may be difficult to find policies that maximize the performance criteria for all agents. So in stochastic games, an equilibrium is 
always looked for every state. This equilibrium is a situation in which no agent, taking the other agents' actions as given, can improve its performance criteria by choosing an alternative action: we find here the definition of the Nash equilibrium (Nash, 1950).

Definition 1 A Nash Equilibrium is a set of strategies (actions) $a^{*}$ such that:

$$
R_{i}\left(a_{i}^{*}, a_{-i}^{*}\right) \geqslant R_{i}\left(a_{i}, a_{-i}^{*}\right) \forall i \in A g, \forall a_{i} \in A_{i}
$$

\subsubsection{Strategic dominance}

When the number of agents is large, it becomes difficult for everyone to consider the entire joint-action space. This may involve a high cost of matrices construction and resolution. To reduce the joint-action set, most research (in the game theory) focused on studying the concepts of plausible solutions. Strategic dominance (Fudenberg and Tirole, 1991; Leyton-Brown and Shoham, 2008) represents one of the most widely used concept, seeking to eliminate actions that are dominated by others actions.

Definition 2 A strategy $a_{i} \in A_{i}$ is said to be strictly dominated if there is another strategy $a^{\prime} \in A_{i}$ such as:

$$
R_{i}\left(a_{i}^{\prime}, a_{-i}\right)>R_{i}\left(a_{i}, a_{-i}\right) \quad \forall a_{-i} \in A_{-i}
$$

Thus a strictly dominated strategy for a player yields a lower expected payoff than at least one other strategy available to the player, regardless of the strategies chosen by everyone else. Obviously, a rational player will never use a strictly dominated strategy. The process can be repeated until strategies are no longer eliminated in this manner. This prediction process on actions, is called "Iterative Elimination of Strictly Dominated Strategies" (IESDS).

Definition 3 For every player $i$, if there is only one solution resulting from the IESDS process, then the game is said to be dominance solvable and the solution is a Nash equilibrium.

However, in many cases, the process ends with a large number of remaining strategies. To further reduce the joint-action space, we could relax the principle of dominance and so include weakly dominated strategies.

Definition 4 A strategy $a_{i} \in A_{i}$ is said to be weakly dominated if there is another strategy $a_{i}^{\prime} \in A_{i}$ such as:

$$
R_{i}\left(a_{i}^{\prime}, a_{-i}\right) \geqslant R_{i}\left(a_{i}, a_{-i}\right) \quad \forall a_{-i} \in A_{-i}
$$

Thus the elimination process would provide more compact matrices and consequently reduce the computation time of the equilibrium. However, this procedure has two major drawbacks: (1) the elimination order may change the final outcome of the game and (2) eliminating weakly dominated strategies, can exclude some Nash equilibria present in the game.

\subsubsection{Best-Response function}

As explained above, the iterative elimination of dominated strategies is a relevant solution, but unreliable for an exact search of equilibrium. Indeed discarding dominated strategies narrows the search for a solution strategy, but does not identify a unique solution. To select a specific strategy requires to introduce the concept of BestResponse.

Definition 5 Given the other players' actions $a_{-i}$, the Best-Response (BR) of the player $i$ is:

$$
B R_{i}: a_{-i} \rightarrow \operatorname{argmax}_{a_{i} \in A_{i}} R_{i}\left(a_{i}, a_{-i}\right)
$$

Thus, the notion of Nash equilibrium can be expressed using the concept of Best-Response:

$\forall i \in A g a_{i}^{*} \in B R_{i}\left(a_{-i}^{*}\right)$

\subsection{Solving stochastic games}

The main works on planning in stochastic games are the ones of Shapley (Shapley, 1953) and Kearns (Kearns et al., 2000). Shapley was the first to propose an algorithm for stochastic games with an infinite horizon for the case of zero-sum games. The FINITEVI algorithm by Kearns et al., generalizes the Shapley's work to generalsum case.

In the same area, the algorithm SGInfiniteVI (Hamila et al., 2010) brought several improvements, including the decentralization and the implementation of the equilibrium selection function (previously regarded as an oracle), in the aim to deal with complex situations, such as the equilibrium multiplicity.

However, the algorithm reaches its limits as the problem size increases. Our objective consists in improving the algorithm SGInfiniteVI by eliminating useless strategies, with the aim to accelerate the computation time, to reduce the memory usage and to plan easily with fewer coordination problems. 


\section{Integration of the dominance procedure and application}

In this section we present the dominance procedure (IEDS) and how we integrate it into the SGInfiniteVI algorithm.

\subsection{The improvement of SGInfiniteVI}

We first present the algorithm 1, which performs the iterative elimination of dominated strategies (introduced in Section 2). The algorithm takes as parameter a matrix $M(s)$ of an arbitrary dimension and returns a matrix $M^{\prime}(s)$ assumed to be smaller. The elimination process is applied iteratively until each player has one remaining strategy, or a number of strategies none of which are weakly dominated. Note that each player will seek to reduce its matrix, not only by eliminating its dominated strategies but also those of others.

The IEDS is incorporated into the algorithm (Algorithm 2, line 8) in the aim to:

- Significantly reduce the matrix size: if after the procedure, there is more than one strategy per player (line 12), the equilibrium selection must be refined to favor a strategy over another one. Thus, Best-Response function is used to find equilibria.

- Directly calculate a Nash equilibrium: this happens when the intersection of all strategies (one per player) forms a Nash equilibrium (line 8).

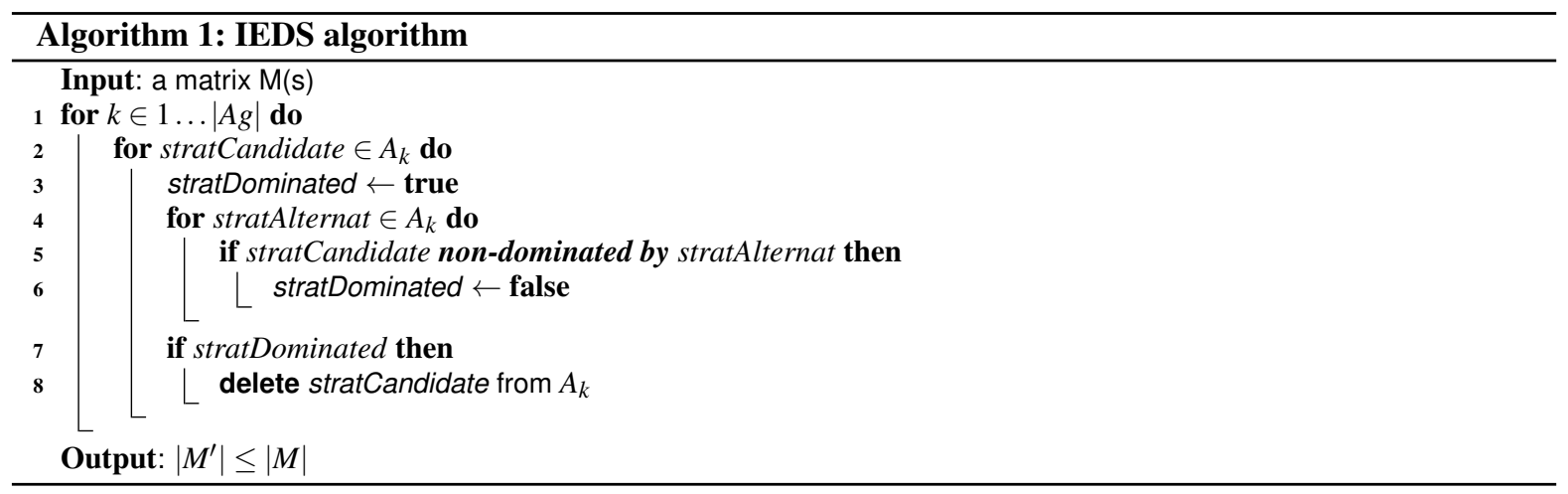

Note that in case of equilibrium multiplicity, we propose two selection functions ${ }^{1}$ : one function maximizes the overall gain $f^{\text {NashMaxTot }}$ and the other maximizes the individual gain $f^{\text {NashMaxSub }}$. If there is no equilibrium then we use the function $f^{\text {ApproxNash }}$ to reach an approximate Nash equilibrium.

Algorithmic complexity: In the search for equilibria, the algorithm is dealing only with a pure Nash equilibrium. It has been proved that whether a game has a "pure Nash equilibrium" is NP-complete (Conitzer and Sandholm, 2008). Therefore, the running time of SGInfiniteVI algorithm is not polynomial in the size of the game matrix (due to the fact that the function $f$ used to compute Nash equilibrium is itself non-polynomial). Moreover, the size of the states space is exponential in the number of agents. The running time of our algorithm is taken to be exponential.

Spatial complexity: Since the agent can only keep the equilibrium values, the total number of required values is: $|S| \times|A g|$. SGInfiniteVI is linear in the number of states and agents ${ }^{2}$. the matrix is composed of $|A g|$ values, making a total of $|A g| \times|A|$ values. Every agent can store a backup matrix after each evaluation of the state $s$, including its own payoff but also the payoffs of the other agents. But it is not necessary, since the agent can only keep the values of the payments coming from the calculated equilibrium. Therefore the total number of required values is: $|S| \times|A g|$. SGInfiniteVI is linear in the number of states and agents ${ }^{3}$.

\footnotetext{
${ }^{1}$ We propose only the functions $f^{\text {NashMaxTot }}$ and $f^{\text {NashMaxSub }}$ because it has been shown that these functions were better than the NashPareto in case of equilibrium multiplicity.

2 the next section will show that this complexity is a worst-case complexity and that in practice a gain in memory can be considered.

${ }^{3}$ the next section will show that this complexity is a worst-case complexity and that in practice a gain in memory can be considered.
} 


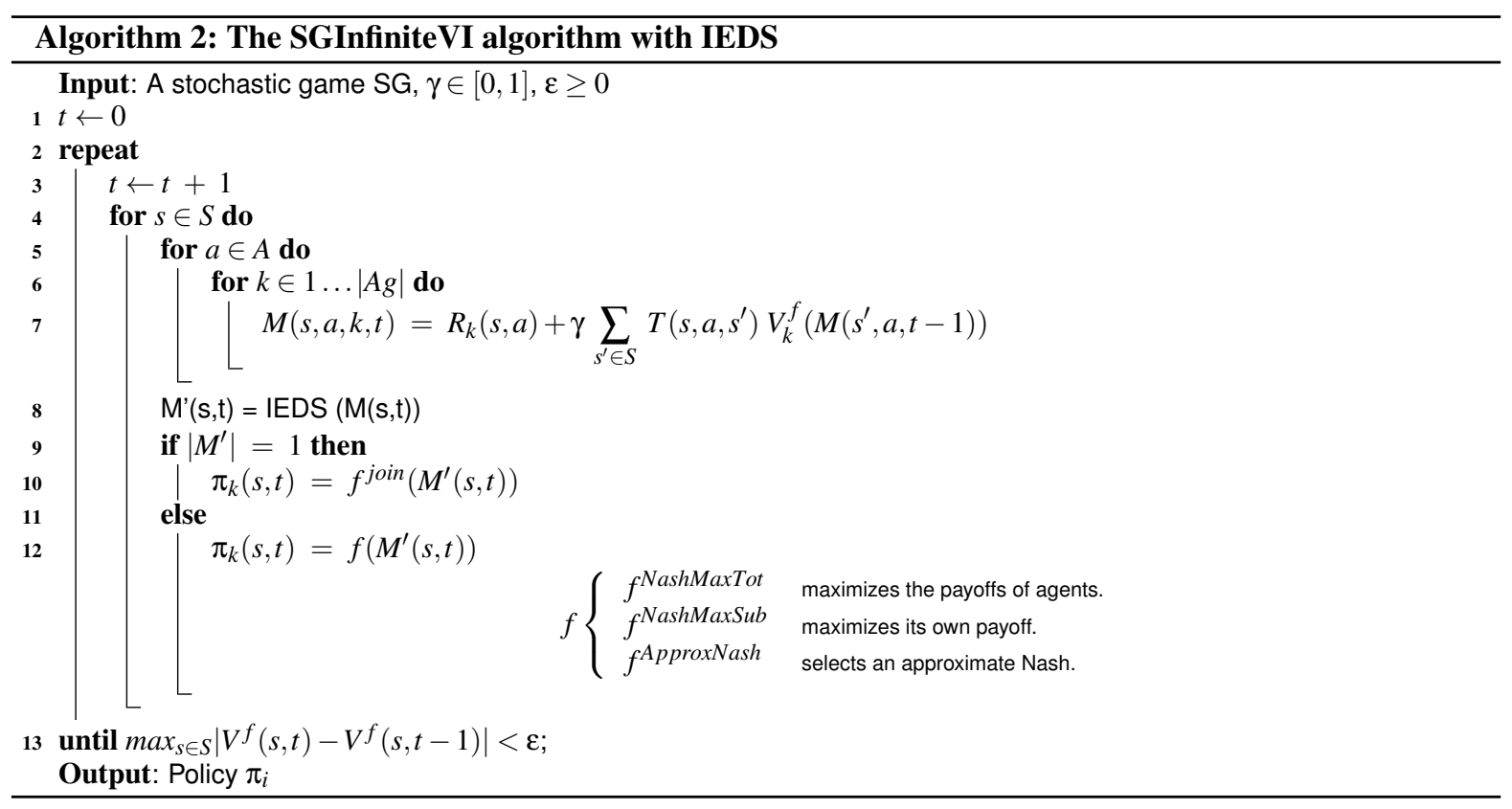

\subsection{Grid-world game}

The example that we choose is similar to the examples from literature, as the "Two-Player Coordination Problem" of $\mathrm{Hu}$ and Wellman (Hu and Wellman, 2003). It is the problem of multi-robot box-pushing (see Figure 1), the game includes robots, objects and a container box. In this example, the objective of the robots is to put all the objects in the box with a minimum number of steps without conflict.

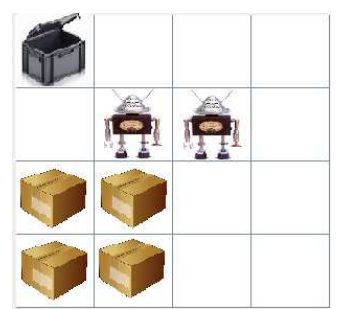

Figure 1: An example of scenario: two robots, four objects and a container box.

The following section intends to validate the improved algorithm on the modeled game.

\section{Validation}

The experiments were performed on 200 policies (one for each agent), with 20,000 tests per policy (changing at each test randomly the agents initial-position). The simulator was implemented in Java language and the experiments were performed on a machine quad-core $2.8 \mathrm{GHz}$ and $4 G B$ of memory.

First, experiments were made to study the effect of IEDS procedure on the used resources. Second, we sought to determine its effect on the agents' behavior.

\subsection{Numerical evaluation}

This section aims to demonstrate empirically the effect of IEDS on the equilibrium computation, the CPU-time and the memory space (depending on the type of eliminated strategies).

\subsubsection{Equilibrium computation}

We wanted to test the algorithm with two different procedures, respectively performing iterative elimination of strictly and weakly dominated strategies. The purpose is to know which of the two procedures would make a 
profit without degrading the quality of the solution. The optimal case of reduction corresponds to one strategy per player (the joint-action forms an equilibrium). We call $p c R e d$ the percentage of the reduction.

The experiments show that for the case of strictly dominated strategies, the percentage of Nash equilibrium found by the $f^{\text {join }}$ function is generally less than $20 \%$. As for the elimination of weakly dominated strategies, the percentage is close to $90 \%$ as shown in Figure 2. The results reflect the ascendancy of the weakly dominated strategies in terms of matrix reduction.

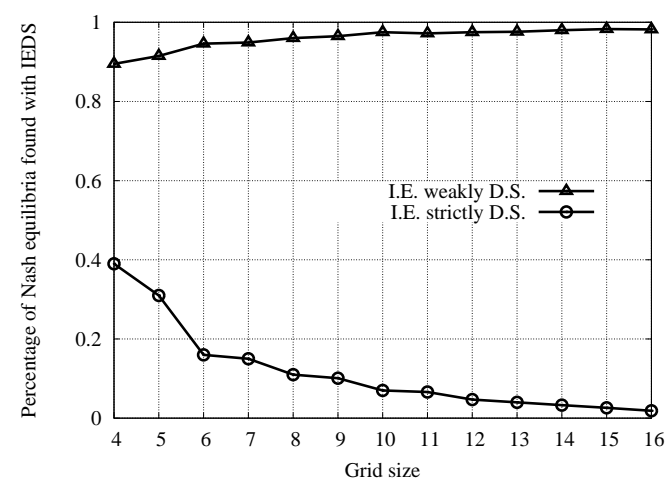

Figure 2: Comparison between IEWDS and IESDS in terms of matrix reduction (with 2 agents, 4 objects and different grid size).

\subsubsection{Evaluation on the computation time}

We compared the computation time used by the algorithm SGInfiniteVI with/without IEDS. The figure 3 shows that the elimination of weakly dominated strategies provides a significant gain in time $G_{t}$, depending on the size of the environment. Nevertheless, the use of strictly dominated strategies does not reduce the computation time but increases it a little. Outside the context of the application, we can say that the reduction of the matrix size

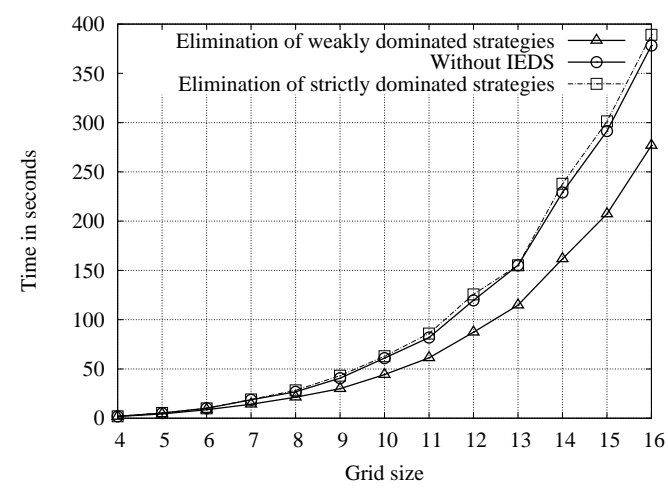

Figure 3: Comparison between IEWDS, IESDS and the original version of the algorithm, in terms of computation time (with 2 agents, 4 objects and different grid size).

is not necessarily synonymous of less computation time. Indeed, the IEDS procedure leads to a gain $g_{t}$ on the exploration time of the matrix, but also entails a computational cost $c_{t}$ in addition to the one of the algorithm, the useful gain is being given by the difference $g_{t}-c_{t}$.

Thus, to obtain a gain in time $G_{t}$ that is perceptible at the general level, the degree of reduction $p c R e d$ must be large enough to generate a gain $g_{t}$ covering $c_{t}$. The parameter $p c R e d$ could be integrated into the algorithm to allow launching (if required) the IEDS procedure.

\subsubsection{Evaluation on memory space}

At this level, the used dominance procedure does not allow to reduce directly the memory usage, but propose an estimation of the gain. Indeed, a gain is possible only when matrices are partially calculated. For example, it is useless to calculate the payments of the other players, when these coincide with a dominated and therefore 
not performed strategy. An estimate of the expected gain can be made from the average number of dominated strategies per player. The scenario is the following one:

1. Each player computes only its own payments. The number of calculated values is: $|A|$,

2. Start the IEDS process,

3. Calculate the payments of the other players when they only correspond to the surviving strategies. The number of new values filled in the matrix is: $\left|A_{-i}\right|$, which makes a total of $|A|+\left|A_{-i}\right|$ by matrix,

4. Find the best-response,

The expected gain per matrix is then:

$$
\begin{aligned}
g_{m} & =(\text { nbrValMat }- \text { nbrValCalc }) / \text { nbrValMat } \\
& =\left((|A| *|A g|)-\left(|A|+\left|A_{-i}\right|\right)\right) /(|A| *|A g|)
\end{aligned}
$$

The total expected gain is:

$$
\begin{aligned}
G_{m} & =g_{m} * \text { pcRed } \\
& =\left((|A| *|A g|)-\left(|A|+\left|A_{-i}\right|\right)\right) * \text { pcRed }
\end{aligned}
$$

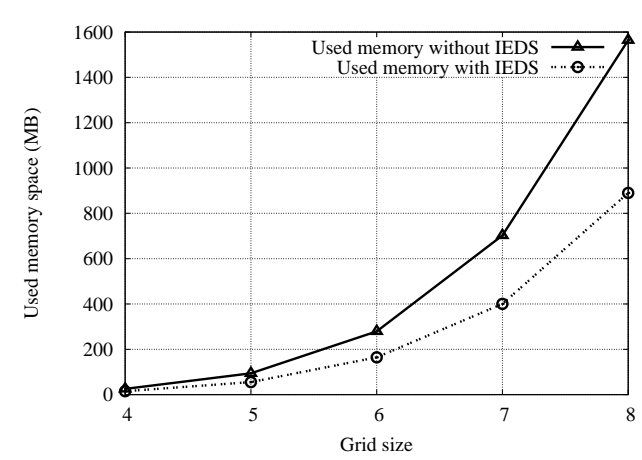

Figure 4: Memory space required to calculate a policy (with 3 agents, 3 objects and different grid size).

The table 1 shows the evolution of the expected gain according to the number of agents and the figure 5 shows in practice the evolution of the expected gain according to the problem size. The empirical analysis of $g_{m}$ and $p c R e d$ leads to an estimated gain $G_{m}$ of about $40 \%$.

\begin{tabular}{|c|c|c|c|c|c|c|}
\hline & $\left|A_{g}\right|$ & $|A|$ & $|M|$ & $g_{m}$ & $p c R e d$ & $G_{m}$ \\
\hline 2 agents & 2 & 25 & 50 & $40 \%$ & $\simeq 90 \%$ & $35 \%$ \\
\hline 3 agents & 3 & 125 & 375 & $60 \%$ & $\simeq 75 \%$ & $45 \%$ \\
\hline 4 agents & 4 & 625 & 2500 & $70 \%$ & $\simeq 53 \%$ & $37 \%$ \\
\hline
\end{tabular}

This will allow considering higher problem sizes, increasing the number of agents, the grid size, etc.

\subsection{Evaluation on the agents' behavior}

To assess the effect of the elimination of dominated strategies ${ }^{4}$ on the agents' behavior; we define three elements of comparison:

- The average number of conflicts: a conflict is considered when agents violate any of the game rules, e.g. an agent moving to a position that is already occupied by another agent.

- The average number of deadlocks: a deadlock occurs when agents are preventing each other's actions forever, waiting for a shared resource.

- The average number of livelocks: a livelock is an endless cycle that prevents the agents from reaching a goal state, and the game from any progress.

\footnotetext{
${ }^{4}$ We consider only weakly dominated strategies, since the elimination of strictly dominated strategies cannot make significant gains.
} 


\subsubsection{Average number of conflicts}

Figure 5 shows that the elimination of dominated strategies leads to an increase in the number of conflicts compared to the original version of the algorithm. Nevertheless, the observed values remain relatively small and can be considered as acceptable in the context of the simulation. For example, for a simulation with a grid size of $12 \times 12$, two agents and four objects, the average number of conflicts is only 0.25 per simulation.

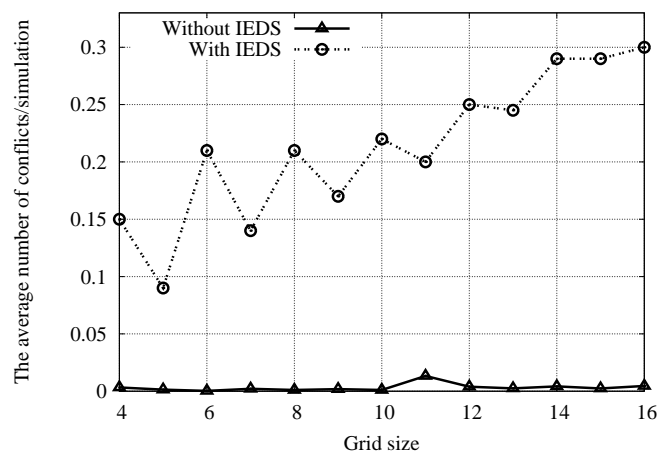

Figure 5: Evaluation according to the average number of conflicts (with 2 agents, 4 objects and different grid size).

\subsubsection{Average number of deadlocks and livelocks}

The figure 6 shows a slight increase in the average number of deadlocks by simulation compared to the original version of the algorithm. The number of deadlocks may reflect a loss in terms of coordination between agents. Indeed, such a situation occurs when the actions performed from distinct Nash equilibria.

\subsubsection{Conclusion of the experimentation}

We found that results show a significant gain in computation time and an opportunity to gain memory space. Intuitively, this gain is not without consequence on the agents' policies. Dominated strategies may affect longterm gains, even though they are considered unnecessary during the elimination process. In addition, adopting the concept of dominance simplifies the decision problem at each stage by getting rid of the equilibrium multiplicity.

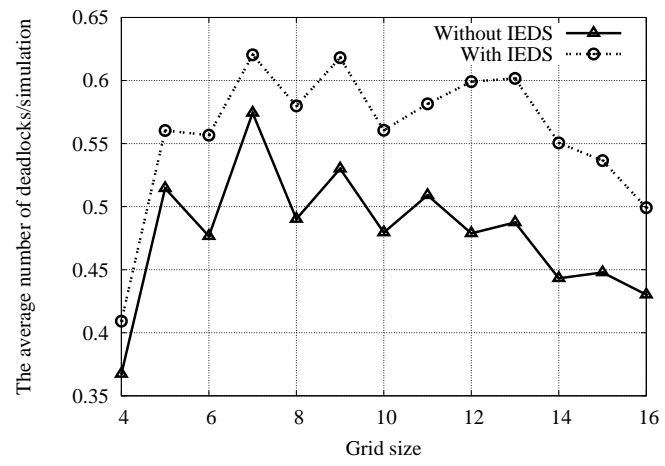

Figure 6: Evaluation according to the average number of deadlocks (with 2 agents, 4 objects and different grid size).

\section{Conclusion}

In the context of coordinating agents, the aim of this work was not only to study the model of stochastic games, but also to propose a planning algorithm based on the dynamic programming and the Nash equilibrium. Our method involves the implementation, the validation and the evaluation on an example of interaction between agents. The concept of strategic dominance has been studied and used in order to improve the SGInfiniteVI algorithm computation time and used memory. The experiments demonstrated that only the elimination of weakly dominated strategies could make a gain in time and memory. 


\section{REFERENCES}

Conitzer, V. and Sandholm, T. (2008). New complexity results about nash equilibria. Games and Economic Behavior, 63(2):621-641.

Fudenberg, D. and Tirole, J. (1991). Game theory. MIT Press, Cambridge, MA.

Hamila, M. A., Grislin-le Strugeon, E., Mandiau, R., and Mouaddib, A.-I. (2010). An algorithm for multi-robot planning: Sginfinitevi. In Proceedings of the 2010 IEEE/WIC/ACM International Conference on Web Intelligence and Intelligent Agent Technology - Volume 02, WI-IAT '10, pages 141-148, Washington, DC, USA. IEEE Computer Society.

Hansen, E. A., Bernstein, D. S., and Zilberstein, S. (2004). Dynamic programming for partially observable stochastic games. In Proceedings of the 19th national conference on Artifical intelligence, AAAI'04, pages 709-715. AAAI Press.

Hu, J. and Wellman, M. (2003). Nash q-learning for general-sum stochastic games. Journal of Machine Learning Research, 4:1039-1069.

Kearns, M., Mansour, Y., and Singh, S. (2000). Fast planning in stochastic games. In In Proc. UAI-2000, pages 309-316. Morgan Kaufmann.

Leyton-Brown, K. and Shoham, Y. (2008). Essentials of game theory: A concise multidisciplinary introduction. Synthesis Lectures on Artificial Intelligence and Machine Learning, 2(1):1-88.

Nash, J. F. (1950). Equilibrium points in n-person games. Proc. of the National Academy of Sciences of the United States of America, 36(1):48-49.

Neumann, J. V. and Morgenstern, O. (1944). Theory of games and economic behavior. Princeton University Press, Princeton. Second edition in 1947, third in 1954.

Puterman, M. (2005). Markov Decision Processes: Discrete Stochastic Dynamic Programming. Wiley-Interscience.

Shapley, L. (1953). Stochastic games. Proc. of the National Academy of Sciences USA, pages 1095-1100.

Shoham, Y., Powers, R., and Grenager, T. (2003). Multi-agent reinforcement learning: a critical survey. Technical report, Stanford University. 